\title{
SPACE-BORNE LASER ALTIMETER GEOLOCATION ERROR ANALYSIS
}

\author{
Yu Wang ${ }^{1.2^{*}}$ Jian Fang ${ }^{1.2}$ Yu $\mathrm{Ai}^{3}$ \\ ${ }^{1}$ State Key Laboratory of Geo-Information Engineering Xi'an, China, 710054 \\ ${ }^{2}$ Xi'an Surveying and Mapping Institute, Xi'an, China, 710054 \\ ${ }^{3}$ Aerors Inc., Xi'an, No. 1 Middle Yanta Road, Xi'an, China, 710054
}

\section{Commission II, WG II/10}

KEY WORDS: Laser altimetry; Error analysis; Footprints; Pointing angle; Range; Geolocation accuracy

\begin{abstract}
:
This paper reviews the development of space-borne laser altimetry technology over the past 40 years. Taking the ICESAT satellite as an example, a rigorous space-borne laser altimeter geolocation model is studied, and an error propagation equation is derived. The influence of the main error sources, such as the platform positioning error, attitude measurement error, pointing angle measurement error and range measurement error, on the geolocation accuracy of the laser spot are analysed by simulated experiments. The reasons for the different influences on geolocation accuracy in different directions are discussed, and to satisfy the accuracy of the laser control point, a design index for each error source is put forward.
\end{abstract}

\section{OVERVIEW}

In 1974, laser altimetry technology was used for the first time in the Apollo Lunar Orbiter. Since then, Japan, China and other countries have also launched research into space-borne laser altimetry. The basic technical parameters of the typical laser altimeter satellites which have been launched to date are listed in Table 1(Wen Hanjiang et al.,2005) (Wang Jianyu et al.,2010) (Han Ling et al.,2016).

Table 1 Basic technical parameters of the typical laser altimeter satellites launched to date

\begin{tabular}{|c|c|c|c|c|c|c|c|c|}
\hline System & MOLA & GLAS & MLA & LOLA & LALT & Change-1 & $\begin{array}{c}\text { Change- } \\
2 .\end{array}$ & $\begin{array}{c}\text { ZY3- } \\
02\end{array}$ \\
\hline Country & $\begin{array}{l}\text { United } \\
\text { States }\end{array}$ & $\begin{array}{l}\text { United } \\
\text { States }\end{array}$ & $\begin{array}{l}\text { United } \\
\text { States }\end{array}$ & $\begin{array}{l}\text { United } \\
\text { States }\end{array}$ & Japan & China & China & China \\
\hline Task & MGS & ICESat & Messenger & LRO & $\begin{array}{l}\text { SELE } \\
\text { NE }\end{array}$ & CE-1 & CE-2 & $\begin{array}{c}\text { ZY3- } \\
02\end{array}$ \\
\hline $\begin{array}{l}\text { Observation } \\
\text { object }\end{array}$ & $\begin{array}{l}\text { Mars } \\
\text { terrain }\end{array}$ & $\begin{array}{l}\text { Earth } \\
\text { ice } \\
\text { sheets }\end{array}$ & $\begin{array}{l}\text { Mercury } \\
\text { terrain }\end{array}$ & $\begin{array}{l}\text { Moon } \\
\text { terrain }\end{array}$ & $\begin{array}{l}\text { Moon } \\
\text { terrain }\end{array}$ & $\begin{array}{l}\text { Moon } \\
\text { terrain }\end{array}$ & $\begin{array}{l}\text { Moon } \\
\text { terrain }\end{array}$ & $\begin{array}{l}\text { Earth } \\
\text { terrain }\end{array}$ \\
\hline Launch date & 1996 & 2003 & 2006 & 2009 & 2007 & 2007 & 2010 & 2016 \\
\hline Orbit altitude $/ \mathrm{km}$ & 400 & 600 & $200 \sim 15000$ & 50 & $50 \sim 150$ & 200 & 100 & 500 \\
\hline $\begin{array}{c}\text { Laser } \\
\text { wavelength/nm }\end{array}$ & 1064 & $\begin{array}{c}532 / 10 \\
64\end{array}$ & 1064 & 1064 & 1064 & 1064 & 1064 & 1064 \\
\hline Laser number & 1 & 3 & 1 & 2 & & 1 & 1 & 2 \\
\hline Pulse width/ns & $6-7$ & $<6$ & 5 & 6 & 17 & $5-7$ & $5-7$ & 6.5 \\
\hline $\begin{array}{c}\text { Repeat } \\
\text { frequency/Hz }\end{array}$ & 10 & 40 & 8 & $28 \times 5$ & 1 & 1 & $1 / 5$ & 2 \\
\hline $\begin{array}{c}\text { Laser divergence } \\
\text { angle/mrad }\end{array}$ & 0.4 & 0.11 & 0.05 & 0.1 & 0.4 & 0.6 & 0.4 & 0.1 \\
\hline
\end{tabular}

The Geoscience Laser Altimeter System (GLAS) instrument was onboard the NASA ICESAT satellite, which was launched by the United States in 2003. GLAS data can be used to determine land and water terrain along the track. Because all levels of GLAS laser altimetry data can be downloaded freely, scholars engaged in laser altimetry research all over the world have favoured GLAS as the research source data.

NASA officials announced the accuracy of the elevation determination results for GLAS, but NASA has not given a complete description of the space-borne laser altimeter system geolocation error model. Fan (Fan Chunpo, et al.,2007) and Zhu (Zhu Jianfeng, et al.,2014) presented a laser spot geolocation model and the main error sources, but the relationship between the geolocation model and the error sources was not analysed. Huang (Huang Wai, et al.,2016) used a simplified model to analyse the precision change of the

*Corresponding author - 2624206727@qq.com 
space-borne laser altimeter under different terrain conditions. Ma(Ma Yue, et al.,2015) used a simplified model to analyse the attitude influence of the laser altimeter system, and Antarctic LiDAR data were used to test the elevation accuracy of GLAS. Tang(Tang Xinmin, et al.,2016) deduced a precise geometric positioning model for the laser altimeter satellite, but they did not analyse the error of the laser altimeter. Other researchers (Ai Jianhua et al.,2015)- (Lori A et al.,2007) have used SRTM airborne LiDAR data to validate the positioning accuracy of GLAS data.

In this paper, we first deduce the geolocation error propagation equation for the laser altimetry spot elevations from a geolocation model of the space-borne laser altimeter. Simulated data are then used to simulate different geolocation accuracies under different design parameters and error conditions. To satisfy the elevation control point precision for optical stereo positioning, a design index for the space-borne laser altimeter and accuracy requirements for the main error sources are proposed.

\section{RIGOROUS GEOLOCATION MODEL FOR THE GLAS SPACE-BORNE LASER ALTIMETER}

A rigorous positioning model for the GLAS spaceborne laser altimeter is shown in Figure1.a and Figure1.b, where $\mathrm{O}_{\text {Laser }}$ is the reference point of laser emission, $P_{G N S S}$ is the Global Navigation Satellite System (GNSS) antenna phase centre, $O_{B o d y}$ is the satellite quality centre and $P_{\text {Ground }}$ is the laser ground footprint. Space-borne GNSS measures the GNSS phase centre position, and the attitude is determined by the sensitive star sensor system in the J2000 coordinate system. To obtain the position and attitude of the laser altimeter when the laser pulse is launched, the GNSS and star sensor data should be passed to the position and attitude of the laser altimeter. Therefore, we need to measure the GNSS phase centre offset $[D x D y D z]^{\mathrm{T}}$ in the satellite body-fixed coordinate system and the coordinate rotation matrix between the star sensor system and the satellite system $R^{B o d y}{ }_{\text {Star }}$. At the same time, the laser altimeter position offset should also be measured between the satellite $[d x d y d z]^{\mathrm{T}}$ and the laser emission angular rotation matrix of the satellite coordinate system.

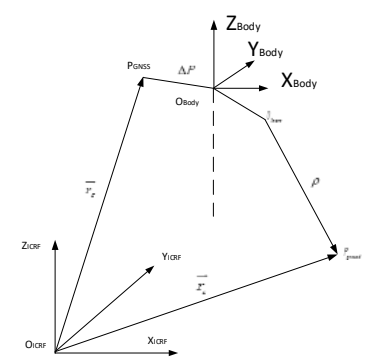

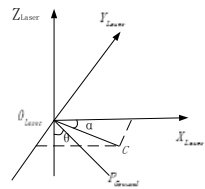

Figure 1. Principle of star-borne laser altimetry.

As shown in Figure 1, assuming that the angle between the laser pointing and altimeter coordinate $\mathrm{Z}$-axis is $\theta$, the angle between $O_{\text {Laser }} C$ (which is the projection of the laser pointing on the XLaserOLaserYLaser plane) and the $\mathrm{X}$-axis is $\alpha$, and $\rho$ is the laser range value, then the coordinates of the laser spot in the satellite coordinate system are:

$$
P_{\text {Ground }}=\left(\begin{array}{c}
\mathrm{dx} \\
\mathrm{dy} \\
\mathrm{dz}
\end{array}\right)+\left(\begin{array}{c}
\rho \sin \theta \cos \alpha \\
\rho \sin \theta \sin \alpha \\
-\rho \cos \theta
\end{array}\right)
$$

Therefore, without considering the effects of atmospheric refraction, the rigorous geolocation model for the space-borne laser altimeter under the International Terrestrial Reference Frame (ITRF) system is:

$\left(\begin{array}{c}X_{P} \\ Y_{P} \\ Z_{P}\end{array}\right)_{\text {ITRF }}=\left(\begin{array}{c}X_{S} \\ Y_{S} \\ Z_{S}\end{array}\right)_{\text {TrRF }}+R_{\text {ICCF }}^{\text {IRFF }} R_{\text {Sar }}^{I C R F} R_{\text {Body }}^{\text {Star }}\left[\left(\begin{array}{c}D \mathrm{x} \\ D \mathrm{y} \\ D \mathrm{z}\end{array}\right)+\left(\begin{array}{c}\mathrm{dx} \\ \mathrm{dy} \\ \mathrm{dz}\end{array}\right)+\left(\begin{array}{c}\rho \sin \theta \cos \alpha \\ \rho \sin \theta \sin \alpha \\ -\rho \cos \theta\end{array}\right)\right]$

Assuming $A=R_{I C R F}^{I T R F} R_{S t a r}^{I C R F} R_{B o d y}^{S t a r}$, we can change (2) to (3):

$\left(\begin{array}{c}X_{P} \\ Y_{P} \\ Z_{P}\end{array}\right)_{\text {ITRF }}=\left(\begin{array}{c}X_{S} \\ Y_{S} \\ Z_{S}\end{array}\right)_{\text {ITRF }}+A \cdot\left[\left(\begin{array}{c}D \mathrm{x} \\ D \mathrm{y} \\ D \mathrm{z}\end{array}\right)+\left(\begin{array}{l}\mathrm{dx} \\ \mathrm{dy} \\ \mathrm{dz}\end{array}\right)+\left(\begin{array}{c}\rho \sin \theta \cos \alpha \\ \rho \sin \theta \sin \alpha \\ -\rho \cos \theta\end{array}\right)\right]$

\section{LASER FOOTPRINT GEOLOCATION ERROR ANALYSIS}

Analysis of (3) shows that the geolocation accuracy of the laser footprint is mainly affected by the following factors. First is the positioning error of the centroid of the satellite $\left(X_{S}, Y_{S}, Z_{S}\right)^{T}$, which is induced by the GNSS receiver positioning error of the satellite and the interpolation calculation. The relationship between the error and the positioning error induced by it is linear, and its influence is limited. Second is the rotation matrix A, which is made up of three parts: the transformation matrix of $R_{I C R F}^{I T R F}$; the rotation matrix between the star sensor coordinate system to the International Celestial Reference Frame (ICRF), $R_{S t a r}^{I C R F}$; and the installation matrix of the star sensor $R_{B o d y}^{\text {Star }}$. The value of $R_{I C R F}^{I T R F}$ is given by the International Earth Rotation Bureau, and its error can be ignored. For the installation matrix $R_{\text {Body }}^{\text {Star }}$, because of its influence on the geolocation error being equal to the attitude error, it is combined with the attitude error.

Third is the installation error of the laser altimeter, which includes the GNSS eccentricity error, the laser reference point eccentricity error, and the laser pointing angle measurement error. The eccentricity errors measured prior to launch are precise, although the values will show a certain change after the satellite is in orbit, but their impact is still relatively small. Due to the range value amplification effect, the laser pointing angle measurement error in a satellite body-fixed system will induce a larger error, so it is analysed in depth.

Fourth is the laser altimeter range error, which includes the system range error and the random error. The system range error is mainly the laser altimeter hardware range error, which can be eliminated by calibration and measurement. The random error mainly includes the transmission error caused by the laser passing through the atmosphere.

Therefore, the analysis of laser footprint error includes four main items: position measurement error, attitude measurement error, pointing angle measurement error and range measurement error, which are shown in (4). 


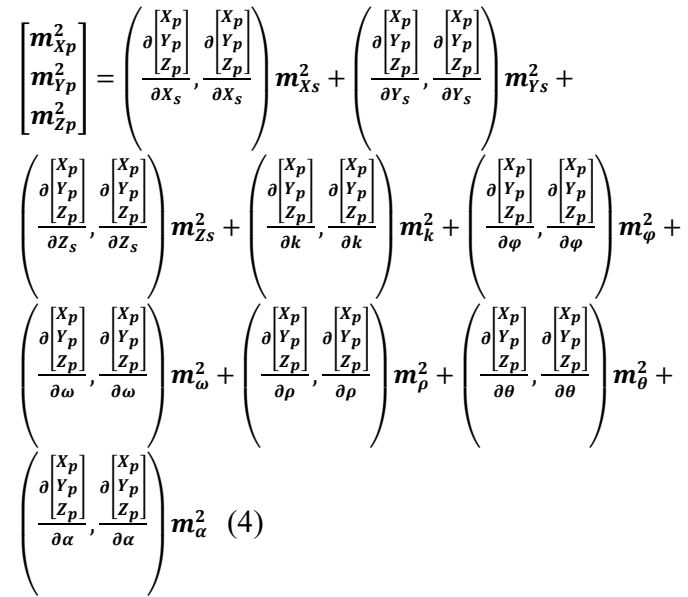

Because of the limited influence of geolocation error, the following analysis concentrates on the influence of attitude measurement error, pointing angle measurement error and range measurement error on the position accuracy of the laser footprint.

\subsection{Attitude measurement error}

The attitude measurement error is mainly induced by the satellite attitude measurement in the orbit of the star sensor and gyro. Attitude measurement error can be divided into pitch $\varphi$, roll wand yaw $\kappa$, which are the angular error components of the three orthogonal directions.

If we define $R_{B o d y}^{I C R F}=R_{S t a r}^{I C R F} \cdot R_{B o d y}^{\text {Star }}=\left[\begin{array}{ccc}a_{1} & a_{2} & a_{3} \\ b_{1} & b_{2} & b_{3} \\ c_{1} & c_{2} & c_{3}\end{array}\right]$, and $R_{B o d y}^{I C R F}$ rotates in order of yaw $\kappa$, pitch $\varphi$ and roll $\omega$, then

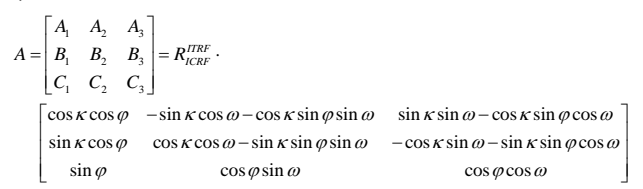

Formula (2) is partially deviated as follows:
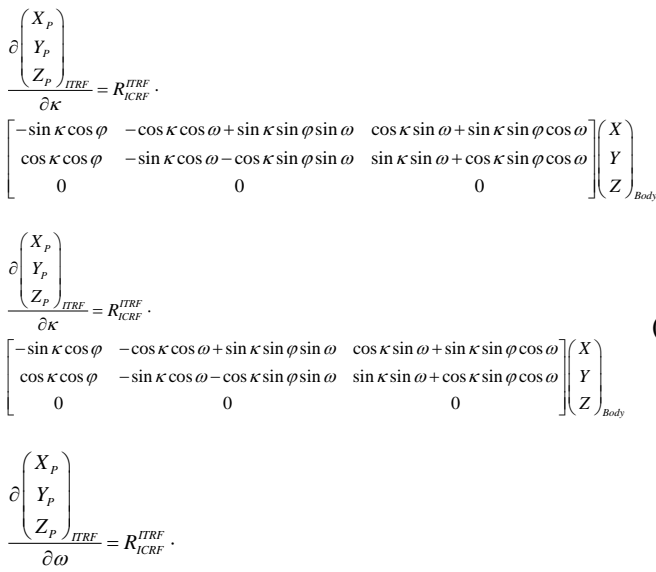

$\left[\begin{array}{ccc}0 & \sin \kappa \sin \omega-\cos \kappa \sin \varphi \cos \omega & \sin \kappa \cos \omega+\cos \kappa \sin \varphi \sin \omega \\ 0 & -\cos \kappa \sin \omega-\sin \kappa \sin \varphi \cos \omega & -\cos \kappa \cos \omega+\sin \kappa \sin \varphi \sin \omega \\ 0 & \cos \varphi \cos \omega & -\cos \varphi \sin \omega\end{array}\right]\left(\begin{array}{l}X \\ Y \\ Z\end{array}\right)_{\text {Bod }}$

In (6), (7) and (8), $\left[\begin{array}{lll}X & Y & Z\end{array}\right]_{B o d y}^{T}$ are the laser footprint coordinates in the satellite coordinate system. The influence of $\operatorname{pitch} \varphi$, roll $\omega$ and yaw $\kappa$ on the laser foot print error in the horizontal and vertical directions can be calculated with the above-mentioned expressions.

\subsection{Pointing angle measurement error}

As mentioned above, the effects of the GNSS and laser altimeter eccentricity error are very limited. The influence of pointing angle error on the geolocation accuracy of the footprint is greatly amplified by the range value. Therefore, in this section, we concentrate on the influence of the pointing angle.

Analysis of (3) shows that the variables in rotation matrix A are only related to the attitude angle and the coordinate system conversion parameters. The angles of $\theta, \alpha$ and the pointing angle are not related to the variable rotation matrix.

The error induced by the angle $\theta$, which is the angle between the laser pointing and the $Z$-axis of the laser altimeter coordinate system, can be expressed as follows:

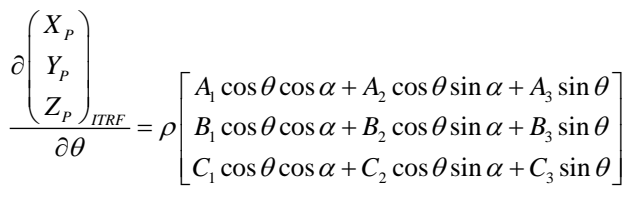

The error induced by the angle $\alpha$, which is the angle between the laser projection in XOY on the horizontal and $\mathrm{X}$-axis, can be expressed as follows:

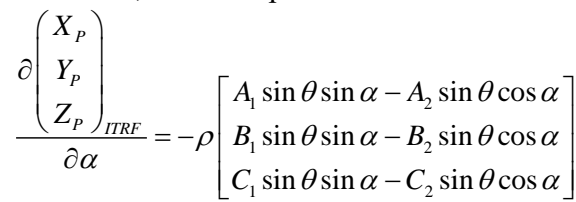

\subsection{Range measurement error}

Although the alignment of the laser is precisely set prior to launch, mechanical forces on lift-off may cause misalignment. The range error mainly includes the time synchronization error, the terrain fluctuation position error, the atmospheric transmission error, etc. The laser footprint position error caused by the range measurement error can be expressed as follows:

$$
\frac{\partial\left(\begin{array}{c}
X_{P} \\
Y_{P} \\
Z_{P}
\end{array}\right)_{I T R F}}{\partial \rho}=\left[\begin{array}{l}
A_{1} \sin \theta \cos \alpha+A_{2} \sin \theta \sin \alpha-A_{3} \cos \theta \\
B_{1} \sin \theta \cos \alpha+B_{2} \sin \theta \sin \alpha-B_{3} \cos \theta \\
C_{1} \sin \theta \cos \alpha+C_{2} \sin \theta \sin \alpha-C_{3} \cos \theta
\end{array}\right]
$$

\section{TEST ANALYSIS}

In order to analyse the influence of the satellite design parameters and calibration errors on the geolocation accuracy of the laser footprint, refer to ICESat and other similar satellite design parameters, analysis condition of formula (4) is simplified with the satellite centroid and the centre offset of the antenna phase are assumed to be 0 , and in the satellite flight process, the satellite posture is more stable, are small angles close to 0 , the attitude angle is set to 0 . Under this condition, formula 4-11 are arranged and three-axis errors are shown as 12 . 


$$
\begin{aligned}
m_{X P}^{2} & =m_{X S}^{2}+\rho^{2} \sin ^{2} \theta \sin ^{2} \alpha m_{k}^{2}+\rho^{2} \cos ^{2} \theta m_{\varphi}^{2}+\sin ^{2} \theta \cos ^{2} \alpha m_{\rho}^{2} \\
& +\rho^{2} \sin ^{2} \theta \sin ^{2} \alpha m_{\alpha}^{2}+\rho^{2} \cos ^{2} \theta \cos ^{2} \alpha m_{\theta}^{2} \\
m_{Y P}^{2} & =m_{Y S}^{2}+\rho^{2} \sin ^{2} \theta \cos ^{2} \alpha m_{\kappa}^{2}+\rho^{2} \cos ^{2} \theta m_{\omega}^{2}+\sin ^{2} \theta \sin ^{2} \alpha m_{\rho}^{2} \\
& +\rho^{2} \sin ^{2} \theta \cos ^{2} \alpha m_{\alpha}^{2}+\rho^{2} \cos ^{2} \theta \sin ^{2} \alpha m_{\theta}^{2} \\
m_{Z P}^{2} & =m_{2 S}^{2}+\rho^{2} \sin ^{2} \theta \cos ^{2} \alpha m_{\varphi}^{2}+\rho^{2} \sin ^{2} \theta \sin ^{2} \alpha m_{\omega}^{2}+\cos ^{2} \theta m_{\rho}^{2} \\
& +\rho^{2} \sin ^{2} \theta m_{\theta}^{2}
\end{aligned}
$$

The influence on the laser footprint geolocation accuracy is analysed with the satellite design parameters, including the flight altitude $\mathrm{H}$, pointing angle $\theta$, platform position error, attitude measurement error, pointing angle measurement error and the range error. In this simulated experiment, the altitude of the satellite flight is $600 \mathrm{~km}$, the pointing angle $\theta$ is $0.3^{\circ}$ and the pointing angle $\alpha$ is $90^{\circ}$. The relevant measurement error values are shown in Table 2.

Table 2 Value of the measurement errors

\begin{tabular}{|c|c|c|c|}
\hline $\begin{array}{c}\text { Position } \\
\text { error (m) }\end{array}$ & $\begin{array}{c}\text { Attitude } \\
\text { error (") }\end{array}$ & $\begin{array}{c}\text { Range } \\
\text { error (m) }\end{array}$ & $\begin{array}{c}\text { Pointing angle } \\
\text { measurement } \\
\text { error (") }\end{array}$ \\
\hline 0.3 & 1 & 0.25 & 1.5 \\
\hline
\end{tabular}

\subsection{Influence of the satellite design parameters on the accuracy of the footprint geolocation}

In the case of the measurement error being constant, the design parameters of flight altitude and pointing angle are changed in this experiment. To investigate the geolocation error changes of the footprint in the $\mathrm{X}, \mathrm{Y}$ and $\mathrm{Z}$ errors of the three axes directions $\mathrm{Mx}, \mathrm{MY}_{\mathrm{Y}}$ and $\mathrm{M}_{\mathrm{Z}}$ and the total position error $\mathrm{M}_{\mathrm{XYZ}}$, the test results are as shown in Table 3 and Table 4. Analysis of the change is shown in Figure 2 and Figure 3.

Table 3 Influence of the flight altitude

\begin{tabular}{cccccc}
\hline $\mathrm{H}(\mathrm{Km})$ & 800 & 600 & 400 & 300 & 200 \\
\hline $\mathrm{M}_{\mathrm{X}}(\mathrm{m})$ & 3.89 & 2.92 & 1.96 & 1.49 & 1.01 \\
$\mathrm{M}_{\mathrm{Y}}(\mathrm{m})$ & 7.00 & 5.25 & 3.51 & 2.64 & 1.77 \\
$\mathrm{M}_{\mathrm{Z}}(\mathrm{m})$ & 0.39 & 0.39 & 0.39 & 0.39 & 0.39 \\
$\mathrm{M}_{\mathrm{XYZ}}(\mathrm{m})$ & 8.02 & 6.02 & 4.04 & 3.05 & 2.08 \\
\hline
\end{tabular}

Table 4 Influence of the pointing angle $\theta$

\begin{tabular}{cccccc}
\hline$\theta\left({ }^{\circ}\right)$ & 0.1 & 0.7 & 1.5 & 2 & 2.5 \\
\hline $\mathrm{M}_{\mathrm{X}}(\mathrm{m})$ & 2.92 & 2.92 & 2.93 & 2.93 & 2.93 \\
$\mathrm{M}_{\mathrm{Y}}(\mathrm{m})$ & 5.25 & 5.25 & 5.25 & 5.25 & 5.25 \\
$\mathrm{M}_{\mathrm{Z}}(\mathrm{m})$ & 0.39 & 0.40 & 0.41 & 0.43 & 0.45 \\
$\mathrm{M}_{\mathrm{XYZ}}(\mathrm{m})$ & 6.02 & 6.02 & 6.02 & 6.03 & 6.03 \\
\hline
\end{tabular}

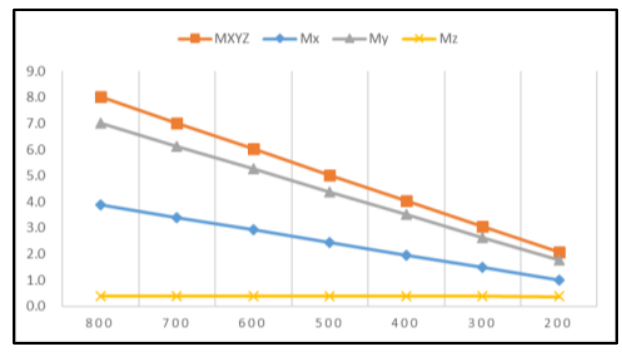

Figure 2. Influence of the flight altitude

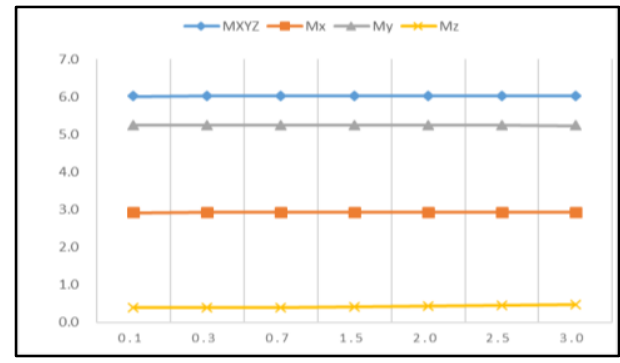

Figure 3. Influence of the pointing angle $\theta$

From Table 3 and Figure 2, it is not difficult to see that under the same error conditions, the geolocation error is reduced when the flight altitude decreases linearly, which suggests that appropriate lowering of the flight altitude could help to reduce the influence of various errors. When the error changes of the three axes directions are compared, the error change in the $\mathrm{X}, \mathrm{Y}$ direction is more drastic, and the error change in the $Z$ direction is relatively gentle. When the satellite altitude changes from $800 \mathrm{~km}$ to $200 \mathrm{~km}$, the $Z$ direction error is reduced to less than $2 \mathrm{~cm}$.

Analysis of (13) can explain the reason for the above phenomenon: the flight altitude is proportional to the range value, and the error of the three directions is amplified with the range value.

When $\mathrm{H}=600 \mathrm{~km}, \theta=0.3^{\circ}$ and $\alpha=90^{\circ}$, (12) can be further translated into:

$$
\begin{aligned}
& m_{x p}^{2}=m_{X s}^{2}+(3141.58)^{2} m_{k}^{2}+(599991.775)^{2} m_{\varphi}^{2}+(3141.578)^{2} m_{\alpha}^{2} \\
& m_{Y_{p}}^{2}=m_{Y_{s}}^{2}+(599991.775)^{2} m_{\omega}^{2}+(0.005)^{2} m_{\rho}^{2}+(599991.775)^{2} m_{\theta}^{2} \\
& m_{z p}^{2}=m_{Z_{s}}^{2}+(3141.578)^{2} m_{\omega}^{2}+(0.999)^{2} m_{\rho}^{2}+(3141.578)^{2} m_{\theta}^{2}
\end{aligned}
$$

It can be seen from (13), compared to the $\mathrm{X}, \mathrm{Y}$ direction, that the error influence factor of the $\mathrm{Z}$ direction is much smaller, which explains the reason why error in the $Z$ direction changes relatively gently with the flight altitude.

From analysis of Table 4 and Figure 3 compared to the flight altitude, the effect of the pointing angle $\theta$ on geolocation error is relatively small. When $\theta$ changes from $0.1^{\circ}$ to $3^{\circ}$, the total geolocation error increases by about $4 \mathrm{~cm}$. The main reason for this phenomenon is that the pointing angle $\theta$ influences the geolocation error with a trigonometric function. The error increases in the $\mathrm{X}$ direction while reducing in the $\mathrm{Y}$ direction. As a result, the influence on the total error is very limited. A similar conclusion can be drawn from analysis of pointing angle $\alpha$.

\subsection{The influence of measurement error on the geolocation accuracy of the footprint}

Among the measurement errors, the effect of the platform position measurement error is the simplest. From (13), its effect on the geolocation accuracy of the footprint is not only linear, but also at a quantitative level, simulation results in Figure 5 also confirm the above conclusion. In the following analysis, we consider the influence of attitude measurement error, range measurement error and pointing angle measurement error.

\section{1) The influence of attitude measurement error}

When the other conditions are unchanged, the value of the attitude measurement error changes from 3 " to 0.2 ", the change of position error of the footprint is Table 5 
and Figure 4, where, with the decrease of the attitude measurement error, the precision of the positioning of the footprint is rapidly improved, especially in the X, Y direction. The reason for this kind of phenomenon appearing is that the posture measurement precision affects the laser launch direction determination accuracy, which influences the plane position accuracy of the footprint. At the same time, from the current laser footprint of $10 \mathrm{~m}$ for the plane geolocation accuracy requirement, and considering a certain margin, the precision of the attitude measurement should be better than $1.5^{\prime \prime}$.

Table 5 Influence of attitude measurement error

\begin{tabular}{cccccc}
\hline Error & 3.0 & 2.0 & 1.5 & 1.0 & 0.2 \\
\hline $\mathrm{M}_{\mathrm{X}}$ & 8.73 & 5.83 & 4.37 & 2.92 & 0.66 \\
$\mathrm{M}_{\mathrm{Y}}$ & 9.76 & 7.28 & 6.18 & 5.25 & 4.41 \\
$\mathrm{M}_{\mathrm{Z}}$ & 0.39 & 0.39 & 0.39 & 0.39 & 0.39 \\
$\mathrm{M}_{\mathrm{XYZ}}$ & 13.10 & 9.33 & 7.58 & 6.02 & 4.48 \\
\hline
\end{tabular}

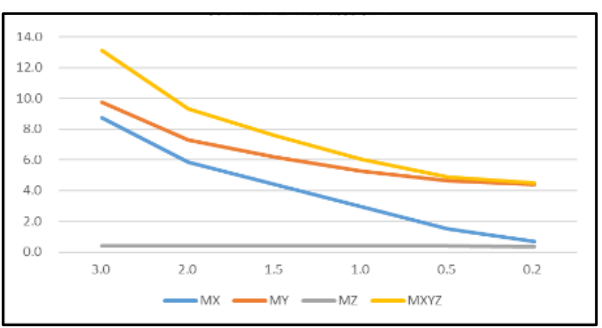

Figure 4. Influence of attitude measurement error

\section{2) The influence of range error}

When the other conditions are unchanged, the range error changes from $1 \mathrm{~m}$ to $0.05 \mathrm{~m}$. The change of the position error is shown in Table 6 and Figure 6, where the range error mainly affects the $\mathrm{Z}$ direction error. Analysis with (13) can draw the same conclusion: the influence of the range error on the plane position error is very small. From the requirement of an elevation position accuracy of $1 \mathrm{~m}$ for the laser control point, the range measurement error should be better than $0.5 \mathrm{~m}$.

Table 6 Influence of range error

\begin{tabular}{cccccc}
\hline Error & 1.00 & 0.50 & 0.25 & 0.20 & 0.05 \\
\hline $\mathrm{M}_{\mathrm{X}}$ & 2.92 & 2.92 & 2.92 & 2.92 & 2.92 \\
$\mathrm{M}_{\mathrm{Y}}$ & 5.25 & 5.25 & 5.25 & 5.25 & 5.25 \\
$\mathrm{M}_{\mathrm{Z}}$ & 1.04 & 0.58 & 0.39 & 0.36 & 0.30 \\
$\mathrm{M}_{\mathrm{XYZ}}$ & 6.10 & 6.04 & 6.02 & 6.02 & 6.01 \\
\hline
\end{tabular}

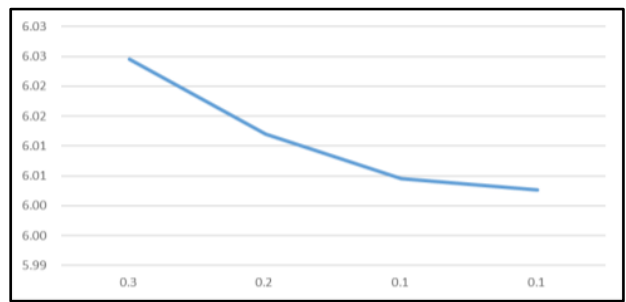

Figure 5. Influence of platform position error

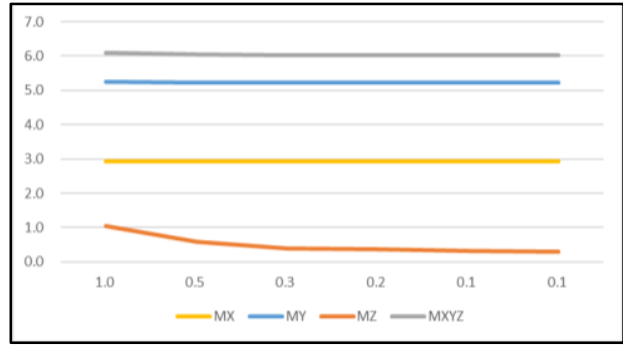

Figure 6. Influence of range error

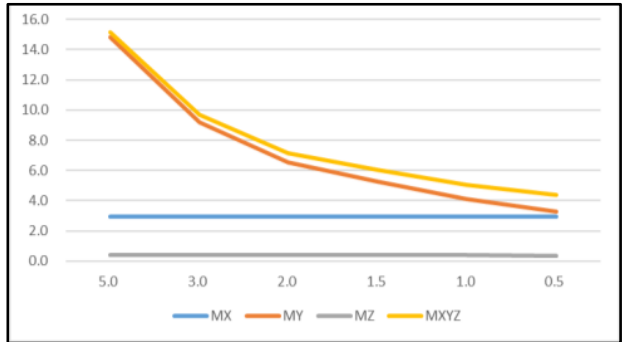

Figure 7. Influence of pointing angle measurement

error

\section{3) The influence of pointing angle measurement} error

The value of pointing angle measurement error changes from $5^{\prime \prime}$ to $0.5^{\prime \prime}$. The changes of the footprint geolocation errors are shown in Table 7 and Figure 7. The influence of the pointing angle measurement error on the geolocation error is like the influence of the attitude measurement error, which mainly affects the accuracy of the plane geolocation error. The geolocation accuracy of the $\mathrm{Z}$ direction is also affected, but the impact is limited. As for the impact in the $\mathrm{X}$ or $\mathrm{Y}$ direction, this depends on the value of $\alpha$. From the requirement of an elevation position accuracy of $10 \mathrm{~m}$ for the current laser control point, the measurement accuracy of the orientation angle should be better than $2 "$.

Table 7 Influence of pointing angle measurement

error

\begin{tabular}{cccccc}
\hline Error & 5.0 & 3.0 & 2.0 & 1.5 & 0.5 \\
\hline $\mathrm{M}_{\mathrm{X}}$ & 2.92 & 2.92 & 2.92 & 2.92 & 2.92 \\
$\mathrm{M}_{\mathrm{Y}}$ & 14.84 & 9.20 & 6.51 & 5.25 & 3.27 \\
$\mathrm{M}_{\mathrm{Z}}$ & 0.40 & 0.39 & 0.39 & 0.39 & 0.39 \\
$\mathrm{M}_{\mathrm{XYZ}}$ & 15.13 & 9.66 & 7.14 & 6.02 & 4.40 \\
\hline
\end{tabular}

\section{CONCLUSION}

In this paper, based on the rigorous geolocation model for the GLAS space-borne laser altimeter, the error propagation equations of the main error sources have been deduced and the influence of various error sources on the geolocation accuracy has been analysed through simulated experiments. The attitude error, pointing angle measurement error and range measurement error all have a great influence on the geolocation accuracy. The attitude measurement error and pointing angle measurement errors mainly affect the accuracy of the plane geolocation of the laser footprint, and the range error is important for the accuracy of elevation 
geolocation.

Analysis from the angle of meeting the design and development requirements of the satellite show that, in the condition that the laser altimeter technology was determined, it would have been advantageous to reduce the altitude of the flight to improve the geolocation accuracy. At a $600 \mathrm{~km}$ orbit altitude, if the requirement of the position accuracy is to meet the plane geolocation accuracy of $10 \mathrm{~m}$ and the elevation position accuracy of $1 \mathrm{~m}$, the laser altimeter attitude measurement accuracy and pointing angle measurement accuracy should be at least better than $1.5 "$. In this paper, the influence of terrain slope on geolocation accuracy has not been taken into account, so some of the index requirements are more lenient. This aspect will be considered in our further research.

\section{REFERENCES:}

Ai Jianhua,2015.SRTM data precision evaluation using ICESAT/GLAS laser altimetry data $[\mathrm{J}]$, Surveying and mapping equipment., 17 (2) ,pp.63-66.

Fan Chunpo, Li Jiancheng, Wang Dan, 2007.ICESAT /GLAS laser footprint geolocation and error analysis. 27 (1), pp.104106.

Han Ling, Tian shijiang, Xie Junfeng, 2016.Development status of calibration technique for space-borne laser altimeter [J]. Spacecraft Recovery and Remote sensing, 12,pp.11-19.

Hieu Duong, Roderik C, Lindenbergh etc.,2009. ICESAT fullwaveform altimetry compared to airborne laser scanning altimetry over the Netherlands [J], IEEE transactions on geoscience and remote sensing, 47(10),pp.3365-3378.

Huang Wai, Li Guo Yuan, Li Shanshan and, etc., 2016.Error analysis of satellite laser altimetry system under different terrain conditions[J].Surveying and Mapping Science, 41 (1) ,pp.44-49.

Lori A, Magruder, Charles E, etc., 2007.ICESAT altimetry data product verification at white sands space harbor[J]. IEEE transactions on geoscience and remote sensing, 45(1),pp.149155

Ma Yue, Yang Fanling, Lu Xiushan, etc.,2015. Elevation error analysis of spaceborne laser altimeter for earth observation [J].Infrared and Laser Engineering, 44 (3) ,pp.1042-1047.

Tang Xinmin, Li Guoyuan, Gao Xiaoming, etc., 2016.The rigorous geometric model of satellite laser altimeter and preliminarily accuracy validation[J], Journal of Surveying and Mapping, 45 (10), pp.1182-1191.

Wang Jianyu, Shu Rong, Chen Weibiao, etc., 2010.Change-1 space-borne laser altimeter. [J]. Chinese science, 40 , pp.10631070.

Wen Hanjiang, Cheng Pengfei, 2005.Introduction to principle of ICESAT/ GLAS laser altimetry and its applications [J]. Surveying and Mapping Science,30, pp.33-35

Zhu Jianfeng, Wang Yu, Hu Yu, etc.,2014. Systematic error analysis and calibration of space-borne laser altimeter [J].Surveying and Mapping Bulletin, (S1) ,pp.137-140. 